\title{
An online Sexual Health and Rehabilitation eClinic (TrueNTH SHAReClinic) for prostate cancer patients: a feasibility study
}

\author{
A. G. Matthew ${ }^{1}$ - L. J. Trachtenberg ${ }^{1} \cdot$ Z. G. Yang ${ }^{1} \cdot$ J. Robinson $^{2} \cdot$ A. Petrella ${ }^{1} \cdot$ D. McLeod ${ }^{3} \cdot$ L. Walker $^{2} \cdot$ R. Wassersug ${ }^{4}$.

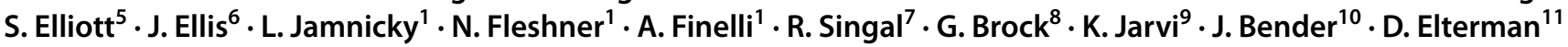

Received: 23 April 2021 / Accepted: 15 August 2021 / Published online: 31 August 2021

(c) The Author(s), under exclusive licence to Springer-Verlag GmbH Germany, part of Springer Nature 2021

\begin{abstract}
Purpose The primary objective was to determine the feasibility of implementing the TrueNTH SHAReClinic as a panCanadian sexual health and rehabilitation intervention for patients treated for localized prostate cancer.

Methods The feasibility study was designed to evaluate the accessibility and acceptability of the intervention. Participants from five institutions across Canada were enrolled to attend one pre-treatment and five follow-up online clinic visits over 1 year following their prostate cancer (PC) treatment.

Results Sixty-five patients were enrolled in the intervention. Website analytics revealed that $71 \%$ completed the intervention in its entirety, including the educational modules, with an additional $10 \%$ completing more than half of the intervention. Five thousand eighty-three views of the educational modules were made along with 654 views of the health library items. Over 1500 messages were exchanged between participants and their sexual health coaches. At 12 months, the intervention received an overall average participant rating of 4.1 out of 5 on a single item satisfaction measure.

Conclusion Results support the TrueNTH SHAReClinic as highly acceptable to participants as defined by intervention adherence and engagement. The TrueNTH SHAReClinic demonstrated promise for being a feasible and potentially resourceefficient approach to effectively improving the sexual well-being of patients after PC treatment.
\end{abstract}

Keywords Prostate cancer $\cdot$ Sexual recovery $\cdot$ Intervention $\cdot$ Web-based $\cdot$ Feasibility

\section{Introduction}

The high prevalence of sexual dysfunction (SD) after prostate cancer (PC) treatment and the lack of effective interventions substantially reduce the quality of life $(\mathrm{QoL})$ of PC patients. Research on men post-radiation therapy and post-radical prostatectomy (RP) indicates that $24-7 \%$ and

A. G. Matthew

andrew.matthew@uhn.ca

1 Department of Surgical Oncology, Princess Margaret Cancer Centre, University Health Network, 700 University Avenue, 6th Floor, Room 6-817, Toronto, ON M5G 1Z6, Canada

2 Department of Oncology, University of Calgary, Calgary, $\mathrm{AB}$, Canada

3 Dalhousie University, Halifax, NS, Canada

4 Cellular and Physiological Sciences, University of British Columbia, Vancouver, BC, Canada

5 Departments of Urologic Sciences, Vancouver Prostate Centre, Vancouver, BC, Canada
$40-90 \%$ suffer SD, respectively [1-5]. On average, $60 \%$ of men experience significant distress in response to SD [6-11].

Research examining the nature of the distress reveals that the significance of SD reaches far beyond the ability to have an erection, and includes low self-esteem, guilt, depression, anxiety, and anger [12,13]. Additionally, patients' distress can negatively impact the patient relationships with their

6 Department of Psychiatry, Sunnybrook Health Sciences Centre, Toronto, ON, Canada

7 Toronto East Health Network Michael Garron Hospital, Toronto, ON, Canada

8 Department of Surgery, Western University, London, ON, Canada

9 Department of Surgery, Mount Sinai Hospital, Toronto, ON, Canada

10 Department of Supportive Care, Princess Margaret Cancer Centre, Toronto, ON, Canada

11 Division of Urology, University of Toronto, Toronto, ON, Canada 
partners $[3,7,14]$. Given the extensive burden of SD on patient and partner's QoL [15-17], there is an existent need for effective, comprehensive treatment for SD.

Traditional biomedical interventions for post-treatment SD focused on penile rehabilitation. Penile rehabilitation is defined as, "the use of any intervention or combination (medication, devices, or actions) with the goal of restoring erectile function to pre-treatment levels" [18], p. 198]. Despite positive penile rehabilitation outcomes in animal models [19], strategies to return men to pre-PC treatment levels of spontaneous erectile functioning have not been successful [19-21]. Consequently, the data is inadequate to support any specific penile rehabilitation regimen [22]. Biopsychosocial interventions have been developed that take a more holistic approach to addressing SD following PC treatment [23]. The biopsychosocial approach encompasses both a biomedical and a psychosocial component. The biomedical component focuses on sexual dysfunction including the return of erectile functioning via pro-erectile therapies; and the psychosocial component focuses on sexual satisfaction, distress, confidence, and intimacy maintenance [24]. Although research examining the effectiveness of biopsychosocial interventions is small in number, studies are beginning to show evidence of benefit for patients [24, 25].

Unfortunately, there are many barriers to accessing and participating in face-to-face clinics. Common practical barriers include resource limitations within treating institutions (e.g., clinic space, human resources, and expertise), geographic distance, and transportation costs in time and money for patients (exacerbated by the COVID-19 pandemic and physical distancing concerns). Currently, programs to help PC patients with SD in Canada are only accessible to those treated at a few comprehensive cancer care centers. Psychosocial barriers can include cancer-related fatigue, social anxiety, and stigma (e.g., around cancer and SD) [26]. These barriers can result in prolonged suffering for patients and highlight the importance of using internet access to provide $\mathrm{SD}$ clinical services that can reach patients outside the clinic.

Internet-delivered interventions address such barriers and can be an important pathway to sexual recovery for men with PC. Recent advances in digital healthcare, combined with the increasing appreciation for the need of sexual health programming in cancer, have established a demand for digital health interventions for SD following PC treatment [27], especially given the current situation with the COVID-19 pandemic.

While PC patients are typically an older cohort, they have been shown to be internet-literate and have interest in interacting with health care providers online [24, 28-31]. Internet-delivered interventions have also been shown to reduce distress among men with PC and their partners [24, 32]. Schover and colleagues [33] found that an intimacy enhancement and psychoeducational intervention was as effective as a brief in-person sex therapy intervention in improving sexual outcomes after PC. Wittmann and colleagues similarly developed a promising web-based intervention for couples coping with side-effects of PC which they evaluated in a randomized controlled trial [24]. However, there are few reports of interventions that improve sexual function following PC treatment and the QoL of couples long-term.

Here we describe an intervention and feasibility study for an innovative online biopsychosocial clinic, the TrueNTH Sexual Health and Rehabilitation eClinic (TrueNTH SHAReClinic) for helping men (and their partners) recover sexual function after PC treatment.

\section{National collaborative}

The TrueNTH SHAReClinic was developed via a national collaboration of Canadian healthcare practitioners and patient/partner advocates. The TrueNTH SHAReClinic adapted and combined features of the Princess Margaret Cancer Centre Prostate Cancer Rehabilitation Clinic (PM-PCRC), the Tom Baker Cancer Centre Prostate Cancer Sexuality and Bladder Rehabilitation Program (TBSBRP), and the Dalhousie Online Sexual Rehabilitation in Prostate Cancer Feasibility Study (D-OSRP).

\section{Theoretical approach}

The TrueNTH SHAReClinic is based on a broad definition of penile rehabilitation focused on applying a systematic pro-erectile aid regimen to assist patients in achieving long-term return of erectile functioning, firm enough for penetration, with or without the use of pro-erectile agents/ devices. We have termed this biomedical rehabilitation approach, erectile rehabilitation [23]. TrueNTH SHAReClinic helps patients find and adapt to the least invasive pro-erectile agent/device that is successful in achieving desired erections. We justify this approach through research demonstrating that, following PC treatment, patients who use pro-erectile agents report better erectile functioning versus those who do not use such agents [20, $21,34]$. However, we also include a psychosocial approach to support couples in maintaining or restoring intimacy after PC treatment. This part of TrueNTH SHAReClinic is predicated on the fact that combining counseling interventions and medical interventions improves patients' adherence and satisfaction with sexual healthcare in cancer populations $[35,36]$, and in particular in post-RP sexual health treatment and outcomes $[37,38]$. 


\section{Study objective}

To evaluate the effectiveness of the TrueNTH SHAReClinic sexual health and rehabilitation intervention, we plan to compare it to (1) best-practice for parallel outcomes for the face-to-face program at the Princess Margaret Cancer Centre's Prostate Cancer Rehabilitation Clinic [23]; (2) empirical evidence extracted from a systematic literature review; and (3) pre-post data at 1 year follow-up versus baseline data. The overall aim, however, of the present study was to evaluate the feasibility of undertaking a single arm, prospective comparative study of the TrueNTH SHAReClinic as a web-based, pan-Canadian, sexual health and rehabilitation intervention for men treated for PC.

\section{Methods}

Participants from five institutions across Canada were enrolled to attend one pre-treatment and five follow-up online clinic visits over 1 year following their PC treatment. The study recruitment sites included Princess Margaret Cancer Centre (ON), the Vancouver General Hospital (BC), the Tom Baker Cancer Centre (AB), the Nova Scotia Health Authority Cancer Care Program (NS), and the Sunnybrook Health Sciences Centre (ON). The study duration was 18 months (6-month recruitment and 12-month follow-up) starting in late 2017. Primary data analysis was performed once all pilot participants had completed the 12-month visit. Feasibility factors were assessed to inform protocol and intervention refinement, and to evaluate the accessibility and acceptability of a future single arm, prospective comparison study of the TrueNTH SHAReClinic.

\section{Sample}

Participation in the TrueNTH SHAReClinic study was limited to patients returning for prostate biopsy results, or patients with a known PC diagnosis. Radiation oncologists and uro-oncologists approached eligible patients during a regularly scheduled clinical appointment (pre-surgery or preradiation). If the patient expressed interest, the site's Study Coordinator provided the patient and his partner (if present) introduction letters and informed consent forms to review.

The study included both single patients and couples. Eligible participants were those diagnosed with localized PC and scheduled for radical prostatectomy (either open or robotic) or radiation treatment (i.e., brachytherapy or external beam) as their first line of treatment. Participants were hormone and/or chemotherapy-naïve; had access to a computer with internet access; and were at least 18 years of age. Partners of men, who met the above criteria and were 18 years of age or older, were encouraged to participate. Couples were excluded from the intervention if the patient or partner lacked English proficiency, had a medical condition that would preclude safe sexual activity, if the patient was not sexually active, was on nitrate therapy, had other contraindicators to phosphodiesterase type 5 inhibitors (PDE5i's), or had received previous treatment for PC.

\section{Study procedures}

Participants were asked to visit the TrueNTH SHAReClinic website to register for the study using the referral code provided to them by the study team. There were no costs to the participant associated with registration or with using the TrueNTH SHAReClinic platform. Patients/couples were paired with sexual health coaches during registration. These coaches were health care professionals; e.g., social workers, nurses, and psychologists, who had taken the TrueNTH SHAReTraining course [39]. The TrueNTH SHAReTraining course is a specialized online course designed to teach participants knowledge and skills in managing sexual health concerns and to build confidence in providing sexual health care to PC patients and their partners. The 12-week online course was limited to 12 participants and involved $2.0 \mathrm{~h}$ per week of interactive online classes, 2 course facilitators, an extensive multi-media syllabus, all over a 12 week period. To date, eight cohorts have completed the course for a total of 86 graduate trainees.

The sexual health coach contacted participants by telephone to introduce TrueNTH SHAReClinic and answer questions about the study. Following this, participants were asked to complete the TrueNTH SHAReClinic education modules at pre-determined scheduled times based on their PC treatment date. Each e-clinic visit was approximately $30 \mathrm{~min}$. If participants missed their e-clinic visit, they received reminder emails. During and at the end of the study, participants were asked to complete a satisfaction questionnaire that included questions specific to accessing and navigating the TrueNTH SHAReClinic platform as well as their interactions with their sexual health coach. [See Fig. 1 for an overview of the study design.]

\section{Intervention}

The TrueNTH SHAReClinic consisted of facilitated webbased clinic e-visits provided to patients and their partners once pre-operatively, and during follow-up appointments at 6 weeks, 10 weeks, 4 months, 6 months, and 12 months post-PC treatment. The e-visit schedule was chosen to reflect critical points of psychosocial and biomedical sexual healthcare after treatment. Those time-points include pre-treatment 


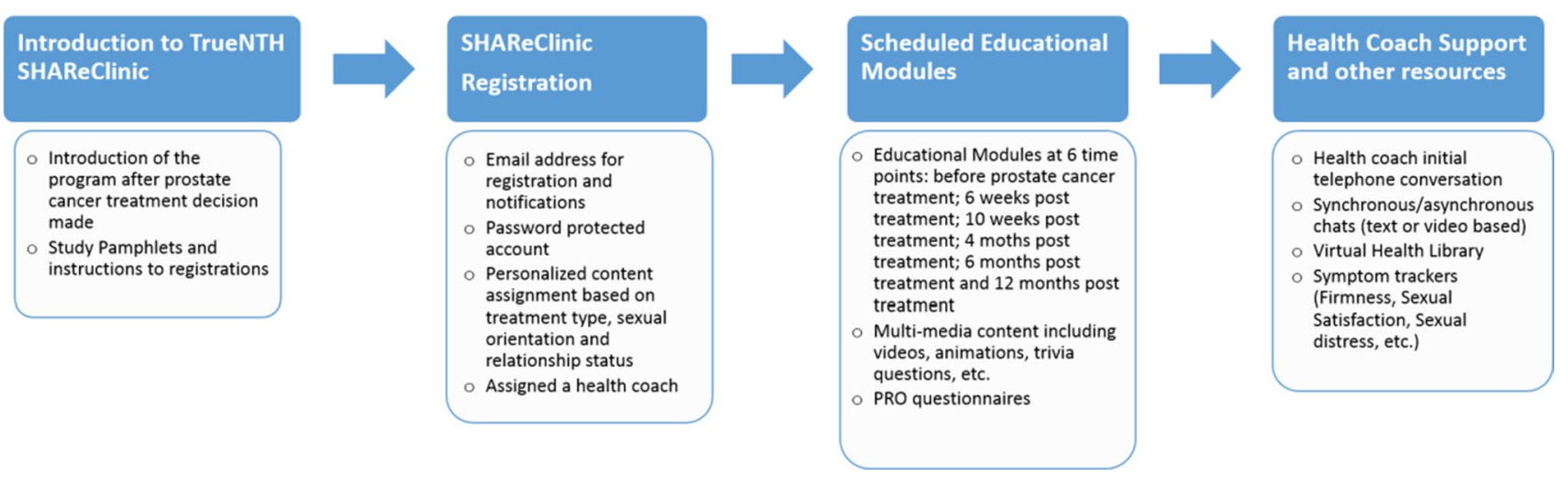

Fig. 1 Overview of study design

to mitigate maladaptive expectations, 6-month follow-up to manage intimacy-related distress and poor adjustment and 1 year follow-up to prioritize acceptance and adaptation to changes in sexual well-being. Similarly, time-points that align with the biomedical erectile rehabilitation algorithm included pre-treatment and 10 weeks follow-up to prioritize early use of pro-erectile agents for long-term penile health, and 1 year follow-up to discuss impact of nerve bundle damage on erectile functioning and potential continued use of pro-erectile agents [40].

The intervention incorporated four components: (1) education modules, (2) facilitation via sexual health coaches, (3) a digital health library, and (4) a tracking and feedback mechanism for both participants and referring physicians.

The education modules comprised of text, interactive activities (e.g., trivia questions), and a total of 28 videos (e.g., with patients, partners, couples, and multidisciplinary healthcare professionals) including biomedical animations. The education modules were tailored to each patient/couple based on demographic and medical information provided during registration including treatment type, relationship status, and sexual orientation. The modules were the main component of each clinic visit. Core topics of the education module included: normalization of sexual health rehabilitation, response expectation, intimacy and passion, challenges to naturalness and spontaneity, adaptation to sexual response changes, performance anxiety, masculinity, partner concerns, sensate focus, mindfulness, adaptation to longterm use of pro-erectile therapy, and enjoyment of nonintercourse sexual activity.

The sexual health coaches facilitated participant experience on the TrueNTH SHAReClinic platform. Using ongoing communication via the platform's private chat feature, the sexual health coaches provided guidance on clinic e-visits and answered questions from participants. The sexual health coaches also received an email alert when a participant posted a question in the chatroom and the coach posted all answers within $48 \mathrm{~h}$. Additionally, for more nuanced care, participants were able to request a check-in telephone call with their sexual health coach at any time during the intervention.

The online clinic also featured a professionally curated, digital, health library including electronic books, relevant leading articles, and videos on sexual health and rehabilitation. Additionally, online trackers were available for participants to receive feedback to monitor their progress. Patient trackers included erectile firmness, sexual satisfaction, sexual distress, intimacy, sexual activity, and medication/ device usage. Trackers were also combined with goal-setting features to encourage patient self-management.

\section{Data analysis}

Results were analyzed through the assessment of system-, practical-, and process-level variables via website onboarding and usage analytics. System-level evaluation included rates of participant recruitment, attrition, completion (e.g., number of participants completing up to and including the 12-month clinic visit), and the number of patients/couples enrolled from each participating institution. The practical level evaluation included technical difficulties (e.g., access to the clinic and materials, completion rate of online questionnaires and the education modules), and an intervention component analysis (e.g., session duration, facilitated topics, educational materials, and communication with sexual health coaches). Process level evaluation included participant's appraisal of their experience with the TrueNTH SHAReClinic.

\section{Results}

\section{Sample}

Sixty-five patients were enrolled in TrueNTH SHAReClinic by the end of the recruitment period. Although the target was to enroll 100 patients, the recruitment period 
was abbreviated due to complex Research and Ethics Board approvals for data-sharing between the "hub" hospital (Princess Margaret Cancer Centre) and the other centers. Consequently, the recruitment periods were as follows: 5 months for Princess Margaret Cancer Centre, 6 weeks for Vancouver General Hospital, 4 weeks for Tom Baker Cancer Centre, 2 weeks for the Nova Scotia Health Authority Cancer Care Program, and 2 weeks for the Sunnybrook Health Sciences. The patient participant distribution included 57 from Ontario, four from British Columbia, two from Alberta, and two from Nova Scotia. In Ontario, TrueNTH SHAReClinic reached participants from 25 different cities and towns outside of the Great Toronto Area. Participating sexual health coaches included five from Ontario, one from British Columbia, one from Alberta, and one from Nova Scotia.

The average age of patients enrolled in the program was 65 years. All participants identified as heterosexual. Ninety percent of participants stated that they were in a romantic relationship, and $86 \%$ reported that they had completed a prostatectomy only (i.e., not additional adjuvant treatment) (see Table 1 for patient demographic information).

Website analytics for all 65 patients revealed that 4 (6.2\%) participants completed and signed consent forms but never entered the program. Of those that entered the program, 46 (71\%) participants completed all of the education modules of the intervention, six (9\%) participants completed greater than $50 \%$, and nine (14\%) completed $20-50 \%$. The education modules used a knowledge build-on model approach requiring participants to complete one module before gaining access to the next one. The completion rates for the questionnaires were $85 \%$ and $71 \%$ at 6- and 12-month follow-up, respectively. Participant engagement in the intervention was substantial with 5083 views of the educational modules, and 654 views of the health library items, and 1235 views of the videos. The most viewed videos included Pro-Erectile Treatment Decision-making, Prostate Anatomy 3D Rotation, and Impact of Radical Prostatectomy on Sexual function. Additionally, 669 messages were sent from participants to sexual health coaches and 899 messages were directed from sexual health coaches to participants via the TrueNTH SHAReClinic secure chatroom. Participants recorded 280 counts of tracker usage. As directed by the intervention, the majority of participants completed the trackers at least once between each SHAReClinic visit. Use of trackers increased after the 10-week visit, suggesting that trackers are more meaningful to participants when they actively reengaging in sexual activity. There were $4318 \log$-in counts by participants and health care providers. Twenty-three percent of log-in counts were from mobile devices (phone or tablet), $74.2 \%$ from computers (desktop or laptop), and $2.5 \%$ from unknown devices.

Shortly after study initiation, five participants complained of technical challenges in registering to the platform. No
Table 1 Patient demographic and medical characteristics

\begin{tabular}{ll}
\hline Patient characteristics & $N(\%)$ \\
\hline Age & \\
$50-59$ & $15(23.1)$ \\
$60-69$ & $28(43.1)$ \\
$70+$ & $22(33.8)$ \\
Ethnicity & \\
White & $42(64.6)$ \\
Black & $5(7.7)$ \\
Latin-American & $2(3.1)$ \\
Asian & $5(7.7)$ \\
Other & $11(16.9)$ \\
Relationship status & \\
Single & $59(90.8)$ \\
Partnered & $6(9.2)$ \\
Type of treatment received & \\
Prostatectomy only & $56(86.2)$ \\
Prostatectomy and radiation therapy & $9(13.8)$ \\
Sexual orientation & \\
Heterosexual & $65(100)$ \\
Gay & 0 \\
Bisexual & 0 \\
Other & 0 \\
\hline
\end{tabular}

further technical difficulties were reported. Following the intervention, participants commented on the efficiency of communication noting that the content of the modules provided for extensive general education while sexual health coach interactions targeted the individualized needs of participants. Overall, at 12 months, participants' satisfaction both in terms of platform usability and patient-coach interaction was rated as 4.1 out 5 .

\section{Discussion}

Biomedical treatments for SD are necessary, but are often insufficient treatment for sexual rehabilitation and recovery after primary PC treatments. SD in patients treated for PC is complex and usually transcends a purely physiological cause. Thus, there exists a need for treatment approaches that account for the multifaceted biopsychosocial concerns that are inherent in SD recovery $[1,23]$. The TrueNTH SHAReClinic proposes an intervention that is based on a biopsychosocial framework. Here we initiated a feasibility study with the ultimate goal of empirically evaluating the efficacy of the TrueNTH SHAReClinic in a large-scale study. Based on the assessment of system, practical, and process level variables, this feasibility study confirms that a full-scale study can be successfully undertaken. 
At the system-level evaluation, only 65 of a planned 100 patients were enrolled in this feasibility study. It is likely that the target number was not reached as a consequence of the aforementioned abbreviated recruitment period. During the actual recruitment period, however, accrual exceeded expectations reach two-thirds of our recruitment goal during a significantly truncated recruitment period across all sites. As well, delays in obtaining inter-institutional data transfer agreements contributed to low participant involvement at other sites. The recruitment in these other centers does however reflect proof of principle of interest and capacity to implement the larger scale TrueNTH SHAReClinic study at those sites.

The study adherence rate was very high with $71 \%$ completing the intervention in its entirety $(80 \%$ completed over half of the intervention). The average adherence rate for online health interventions is around 50\% for an average length of 16.3 weeks of follow-up [41]. Literature suggests that the longer the intervention, the lower the adherence rate $33 \%$ for online lifestyle change interventions with an average length is 30 weeks) [41]. For an intervention like TrueNTH SHAReClinic, with a follow-up period of 12 months, the clinic achieved an exceptionally high adherence rate. We surmise that the elevated completion rate is due to the following: (1) a highly motivated patient population (given the extent of SD and related distress); (2) the systematic use of automated email reminders to patients and sexual health coaches; and (3) the personalized engagement and encouragement of sexual health coaches.

Practical level evaluation revealed some initial difficulty for five participants in registering to the platform. We learned that registration must involve a simple, intuitive process that can be completed in a brief time period. It appears that participant tolerance for registration complexity is very low and can result in abandonment of the process. Website analysis supported successful engagement by participants in all aspects of the TrueNTH SHAReClinic platform including education modules, virtual library, trackers, chatroom, and online questionnaires.

Finally, at the process level, participants reported a high degree of satisfaction in terms of platform usability and patient-coach interaction. Moreover, the hundreds of chatbased interactions underscore the benefit of participantcoach communication, yet coaches reported that the time spent assisting participants was not burdensome and was estimated to average approximately $2-2.5 \mathrm{~h}$ per patient for the full 12 months of the program. As well, mobile devices accounted for one quarter of log-in counts indicating that many participants utilized the platform remotely via tablets and phones. This was similarly true for coaches accessing the platform remotely. These findings support the potential for TrueNTH SHAReClinic as an effective and efficient care provision model in the face of the highly resource burdened healthcare system in Canada.

\section{Conclusion}

Our results support that TrueNTH SHAReClinic is highly acceptable to participants as defined by intervention adherence and engagement. Similarly, TrueNTH SHAReClinic demonstrates promise for being a feasible, knowledge driven, and potentially resource-efficient approach to improving QoL and the sexual wellbeing of patients after PC treatment. Overall, the data presented justifies going forward with the larger comparative study to assess efficacy.

Acknowledgements We thank the health coaches who took part in the study (not including authors): Meaghan Ferguson and Reanne Booker.

Author contribution All authors contributed to the study conception and design. Intervention content was developed by a multi-disciplinary team inclusive of all authors excepting Z.G. Yang, and J. Bender. The intervention transition to a multi-modal and interactive online platform was led by A.G. Matthew and Z.G. Yang. Study design integrity and adherence to protocol was performed by A.G. Matthew, J. Bender, and Z.G. Yang. Data analysis was performed by Z.G. Yang, J. Bender, and D. Elterman. The first draft of the manuscript was written by L.J. Trachtenberg and A.G. Matthew and all authors commented on previous versions of the manuscript. All authors read and approved the final manuscript.

Funding We would also like to recognize the Movember Foundation in collaboration with Prostate Cancer Canada for funding this project.

Data availability All data and material will be made available for audit of results in order to ensure full transparency.

\section{Declarations}

Ethics approval The study protocol was approved by the UHN Research Ethics Board (REB) as well as the institutional review boards of all participating institutions. The procedures used in this study adhere to the tenets of the Declaration of Helsinki.

Consent to participate Informed consent was obtained from all individual participants included in the study.

Consent for publication Within the informed consent process all individual participants consented to the publication of the study.

Competing interests The authors declare no competing interests.

\section{References}

1. Chung E, Brock G (2013) Sexual rehabilitation and cancer survivorship: a state of art review of current literature and management strategies in male sexual dysfunction among prostate cancer survivors. J Sex Med 10(Suppl 1):102-111. https://doi.org/10.1111/j. 1743-6109.2012.03005.x 
2. van der Wielen GJ, Mulhall JP, Incrocci L (2007) Erectile dysfunction after radiotherapy for prostate cancer and radiation dose to the penile structures: a critical review. Radiother Oncol 84(2):107-113. https://doi.org/10.1016/j.radonc.2007.07.018

3. Tanner T, Galbraith M, Hays L (2011) From a woman's perspective: life as a partner of a prostate cancer survivor. J Midwifery Womens Health 56(2):154-160. https://doi.org/10.1111/j.15422011.2010.00017.x

4. Benson CR, Serefoglu EC, Hellstrom WJG (2012) Sexual dysfunction following radical prostatectomy. J Androl 33(6):11431154. https://doi.org/10.2164/jandrol.112.016790

5. Donovan JL, Hamdy FC, Lane JA, Mason M, Metcalfe C, Walsh E, Blazeby JM, Peters TJ, Holding P, Bonnington S et al (2016) Patient-reported outcomes after monitoring, surgery, or radiotherapy for prostate cancer. N Engl J Med 375(15):1425-1437. https://doi.org/10.1056/nejmoa1606221

6. Cooperberg MR, Koppie TM, Lubeck DP, Ye J, Grossfeld GD, Mehta SS, Carroll PR, CaPSURE, (2003) How potent is potent? Evaluation of sexual function and bother in men who report potency after treatment for prostate cancer: data from CaPSURE. Urology 61(1):190-196. https://doi.org/10.1016/s0090-4295(02) 02118-0

7. Lilleby W, Fosså SD, Waehre HR, Olsen DR (1999) Long-term morbidity and quality of life in patients with localized prostate cancer undergoing definitive radiotherapy or radical prostatectomy. Int J Radiat Oncol Biol Phys 43(4):735-743. https://doi. org/10.1016/s0360-3016(98)00475-1

8. Kao TC, Cruess DF, Garner D, Foley J, Seay T, Friedrichs P, Thrasher JB, Mooneyhan RD, McLeod DG, Moul JW (2000) Multicenter patient self-reporting questionnaire on impotence, incontinence and stricture after radical prostatectomy. J Urol 163(3):858-864. https://doi.org/10.1016/s0022-5347(05)67819-6

9. Mirone V, Imbimbo C, Palmieri A, Longo N, Fusco F (2003) Erectile dysfunction after surgical treatment: erectile dysfunction after surgical treatment. Int J Androl 26(3):137-140. https://doi. org/10.1046/j.1365-2605.2003.00384.x

10. Matthew AG, Goldman A, Trachtenberg J, Robinson J, Horsburgh S, Currie K, Ritvo P (2005) Sexual dysfunction after radical prostatectomy: prevalence, treatments, restricted use of treatments and distress. J Urol 174(6):2105-2110. https://doi.org/10.1097/01.ju. 0000181206.16447.e2

11. Arai Y, Okubo K, Aoki Y, Maekawa S, Okada T, Maeda H, Ogawa O, Kato T (1999) Patient-reported quality of life after radical prostatectomy for prostate cancer. Int J Urol 6(2):78-86. https://doi. org/10.1046/j.1442-2042.1999.00629.x

12. Pedersen KV, Carlsson P, Rahmquist M, Varenhorst E (1993) Quality of life after radical retropubic prostatectomy for carcinoma of the prostate. Eur Urol 24(1):7-11. https://doi.org/10. $1159 / 000474254$

13. Helgason AR, Adolfsson J, Dickman P, Arver S, Fredrikson M, Steineck G (1997) Factors associated with waning sexual function among elderly men and prostate cancer patients. J Urol 158(1):155-159. https://doi.org/10.1097/00005392-19970 7000-00050

14. Bates TS, Wright MP, Gillatt DA (1998) Prevalence and impact of incontinence and impotence following total prostatectomy assessed anonymously by the ICS-male questionnaire. Eur Urol 33(2):165-169. https://doi.org/10.1159/000019549

15. Meyer J-P, Gillatt DA, Lockyer R, Macdonagh R (2003) The effect of erectile dysfunction on the quality of life of men after radical prostatectomy. BJU Int 92(9):929-931. https://doi.org/10.1111/j. 1464-410x.2003.04530.x

16. Manne SL (1999) Intrusive thoughts and psychological distress among cancer patients: the role of spouse avoidance and criticism. J Consult Clin Psychol 67(4):539-546. https://doi.org/10.1037// 0022-006x.67.4.539
17. Steginga SK, Occhipinti S, Dunn J, Gardiner RA, Heathcote P, Yaxley J (2001) The supportive care needs of men with prostate cancer (2000). Psychooncology 10(1):66-75. https://doi. org/10.1002/1099-1611(200101/02)10:1\%3c66::aid-pon493\% 3e3.3.co; $2-\mathrm{q}$

18. Mulhall JP, Bivalacqua TJ, Becher EF (2013) Standard operating procedure for the preservation of erectile function outcomes after radical prostatectomy. J Sex Med 10(1):195-203. https:// doi.org/10.1111/j.1743-6109.2012.02885.x

19. Fode M, Ohl DA, Ralph D, Sønksen J (2013) Penile rehabilitation after radical prostatectomy: what the evidence really says: penile rehabilitation after RP. BJU Int 112(7):998-1008. https:// doi.org/10.1111/bju.12228

20. Montorsi F, Brock G, Lee J, Shapiro J, Van Poppel H, Graefen M, Stief C (2008) Effect of nightly versus on-demand vardenafil on recovery of erectile function in men following bilateral nerve-sparing radical prostatectomy. Eur Urol 54(4):924-931. https://doi.org/10.1016/j.eururo.2008.06.083

21. Montorsi F, Brock G, Stolzenburg J-U, Mulhall J, Moncada I, Patel HRH, Chevallier D, Krajka K, Henneges C, Dickson R et al (2014) Effects of tadalafil treatment on erectile function recovery following bilateral nerve-sparing radical prostatectomy: a randomised placebo-controlled study (REACTT). Eur Urol 65(3):587-596. https://doi.org/10.1016/j.eururo.2013.09. 051

22. Salonia A, Adaikan G, Buvat J, Carrier S, El-Meliegy A, Hatzimouratidis K, McCullough A, Morgentaler A, Torres LO, Khera M (2017) Sexual rehabilitation after treatment for prostate cancer-part 2: recommendations from the Fourth International Consultation for Sexual Medicine (ICSM 2015). J Sex Med 14(3):297-315. https://doi.org/10.1016/j.jsxm.2016.11.324

23. Matthew A, Lutzky-Cohen N, Jamnicky L, Currie K, Gentile A, Mina DS, Fleshner N, Finelli A, Hamilton R, Kulkarni G et al (2018) The Prostate Cancer Rehabilitation Clinic: a biopsychosocial clinic for sexual dysfunction after radical prostatectomy. Curr Oncol 25(6):393-402. https://doi.org/10.3747/co.25.4111

24. Wittmann D, Mehta A, Northouse L, Dunn R, Braun T, Duby A, An L, Arab L, Bangs R, Bober S et al (2017) TrueNTH sexual recovery study protocol: a multi-institutional collaborative approach to developing and testing a web-based intervention for couples coping with the side-effects of prostate cancer treatment in a randomized controlled trial. BMC Cancer 17(1):664. https:// doi.org/10.1186/s12885-017-3652-3

25. Walker LM, Hampton AJ, Wassersug RJ, Thomas BC, Robinson JW (2013) Androgen Deprivation Therapy and maintenance of intimacy: a randomized controlled pilot study of an educational intervention for patients and their partners. Contemp Clin Trials 34(2):227-231. https://doi.org/10.1016/j.cct.2012.11.007

26. Trachtenberg L, Wong J, Rennie H, McLeod D, Leung Y, Warner E, Esplen MJ (2020) Feasibility and acceptability of i-Restoring Body Image after Cancer (i-ReBIC): a pilot trial for female cancer survivors. Psychooncology 29(4):639-646. https://doi.org/10. 1002/pon.5288

27. Matthew AG, Yang ZG (2020) Online interventions for sexual health in cancer. Curr Opin Support Palliat Care 14(1):80-86. https://doi.org/10.1097/SPC.0000000000000477

28. Bender JL, Feldman-Stewart D, Tong C, Lee K, Brundage M, Pai H, Robinson J, Panzarella T (2019) Health-related internet use among men with prostate cancer in Canada: cancer registry survey study. J Med Internet Res 21(11):e14241. https://doi.org/10.2196/ 14241

29. Gustafson DH, Hawkins R, McTavish F, Pingree S, Chen WC, Volrathongchai K, Stengle W, Stewart JA, Serlin RC (2008) Internet-based interactive support for cancer patients: are integrated systems better? J Commun 58(2):238-257. https://doi.org/10. $1111 /$ j.1460-2466.2008.00383.x 
30. Crabb RM, Rafie S, Weingardt KR (2012) Health-related internet use in older primary care patients. Gerontology 58(2):164-170. https://doi.org/10.1159/000329340

31. Lu H-Y, Shaw BR, Gustafson DH (2011) Online health consultation: examining uses of an interactive cancer communication tool by low-income women with breast cancer. Int J Med Inform 80(7):518-528. https://doi.org/10.1016/j.ijmedinf.2011.03.011

32. Northouse L, Schafenacker A, Barr KLC, Katapodi M, Yoon H, Brittain K, Song L, Ronis DL, An L (2014) A tailored Web-based psychoeducational intervention for cancer patients and their family caregivers. Cancer Nurs 37(5):321-330. https://doi.org/10. 1097/NCC.0000000000000159

33. Schover LR, Canada AL, Yuan Y, Sui D, Neese L, Jenkins R, Rhodes MM (2012) A randomized trial of internet-based versus traditional sexual counseling for couples after localized prostate cancer treatment: CAREss Counseling Program. Cancer 118(2):500-509. https://doi.org/10.1002/cncr.26308

34. McCullough AR, Levine LA, Padma-Nathan H (2008) Return of nocturnal erections and erectile function after bilateral nervesparing radical prostatectomy in men treated nightly with sildenafil citrate: subanalysis of a longitudinal randomized double-blind placebo-controlled trial. J Sex Med 5(2):476-484. https://doi.org/ 10.1111/j.1743-6109.2007.00700.x

35. Walker LM, Sears CS, Booker R, Doll C, Glaze S, Phan T, Brennan K, Millman RD, Robinson JW (2021) Development, implementation, and evaluation of a multidisciplinary oncology sexual health clinic in a Canadian cancer care setting. J Cancer Surviv 1-12. https://doi.org/10.1007/s11764-020-00967-8

36. Barbera L, Fitch M, Adams L, Doyle C, DasGupta T, Blake J (2011) Improving care for women after gynecological cancer: the development of a sexuality clinic. Menopause 18(12):1327-1333. https://doi.org/10.1097/gme.0b013e31821f598c
37. Davison BJ, Elliott S, Ekland M, Griffin S, Wiens K (2005) Development and evaluation of a prostate sexual rehabilitation clinic: a pilot project. BJU Int 96(9):1360-1364. https://doi.org/10.1111/j. 1464-410X.2005.05833.x

38. Titta M, Tavolini IM, Dal Moro F, Cisternino A, Bassi P (2006) Sexual counseling improved erectile rehabilitation after nonnerve-sparing radical retropubic prostatectomy or cystectomyresults of a randomized prospective study. J Sex Med 3(2):267273. https://doi.org/10.1111/j.1743-6109.2006.00219.x

39. Petrella AR, Robinson J, Osqui L et al (2017) Sexual health and rehabilitation eTraining (SHAReTraining) \& eClinic (SHAReClinic): A Movember TrueNth Canadian Solution. International Conference for Research on Non-Pharmacological Interventions; Toulouse, France.

40. Elterman DS, Petrella AR, Walker LM, Van Asseldonk B, Jamnicky L, Brock GB, Elliott S, Finelli A, Gajewski JB, Jarvi KA et al (2018) Canadian consensus algorithm for erectile rehabilitation following prostate cancer treatment. Can Urol Assoc J 13(8):239-245. https://doi.org/10.5489/cuaj.5653

41. Kelders SM, Kok RN, Ossebaard HC, Van Gemert-Pijnen JEWC (2012) Persuasive system design does matter: a systematic review of adherence to web-based interventions. J Med Internet Res 14(6):e152. https://doi.org/10.2196/jmir.2104

Publisher's note Springer Nature remains neutral with regard to jurisdictional claims in published maps and institutional affiliations. 